\title{
Femmes entre résistance, révolte et affirmation : pour une ré-appropriation identitaire chez Marie Ndiaye
}

\author{
Christian Mbarga \\ Université Saint-Thomas
}

La Sorcière est bâti sur la même thématique qui sous-tend l'œuvre de l'écrivaine: la dynamique des relations de famille, comme dans une grande partie de ses romans tels que En famille, Un temps de saison, Quant au riche avenir, La femme changée en bûche, pour ne citer que ceux-là. Pour nous, il s'agit d'un palimpseste sur lequel Marie Ndiaye réécrit différentes facettes de la même histoire, palimpseste comportant inévitablement une certaine constance de thèmes: l'abandon, 
l'identité, l'exclusion, la disparition. En effet, La Sorcière présente une constellation de couples dysfonctionnels évoluant dans des milieux d'une singularité déprimante, conditions propices à un dénouement apparemment prévisible pour tout lecteur averti, le chemin emprunté pour y aboutir, lui, restant imprévisible. Un couple peut-il survivre à des tensions et à des pratiques qui vont à l'encontre des croyances et valeurs de l'un des époux? Cela résume La Sorcière. Cet article étudiera la manière dont l'écrivaine renforce l'identité féminine, en analysant les outils qu'elle place entre les mains des femmes, notamment la subversion, la violence, seuls moyens efficaces dans la quête de la liberté et du bonheur. Le cadre familial dans lequel évoluent les personnages de ce roman étant important, son introduction dans la partie suivante s'avère ainsi indispensable.

Dans ce roman, plusieurs années de mariage et de vie commune ont finalement raison de couples apparemment solides. Le même schéma se répète : en raison des tensions avec sa femme et, parfois, avec ses enfants quand il s'agit de filles, le mari devient un intrus dans sa propre maison. Envahi d'une frayeur vis-à-vis de sa femme, rempli d'une angoisse intolérable $\mathrm{au}$ point de faire de lui un aliéné, poussé à bout par l'atmosphère dans son foyer, le mari s'enfuit, abandonnant sa famille pour refaire sa vie ailleurs. La description que Lucie, le personnage homodiégétique, fait de son foyer, sans vouloir conforter le comportement de l'homme, abonde néanmoins dans le même sens : une « oppressante atmosphère [...] plutôt disharmonieuse » y règne (p. 16). Le foyer, le centre nerveux de la famille, n'est plus un havre de repos et de félicité où le couple et la famille tout entière se ressource. La maison familiale est devenue plutôt le lieu de misères, de tribulations et de 
frustrations en tous genres pour ses membres, ce qui entraînera immanquablement la dissolution de la famille. En effet, plusieurs événements spécifiques participent de cette dissolution quasi inéluctable des couples, qu'il s'agisse du couple de Lucie, personnage tourmenté à l'identité précaire ${ }^{1}$, et Pierrot son mari, ou de celui d'Isabelle, voisine et amie de Lucie, de Mme Matin ou encore de la mère de Lucie. En effet, une fois que ses deux jumelles avaient atteint l'âge de douze ans, Lucie les initie « aux mystérieux pouvoirs » (p. 9) dans son sous-sol et en moins d'un an, elles passent brillamment leur première épreuve en pleurant des larmes de sang (p. 12), signe précurseur de leurs grands pouvoirs en sorcellerie. Lucie, heureuse de voir le progrès rapide de ses filles, craint néanmoins que son mari Pierre ne ressente envers leurs filles récemment initiées la même "irrépressible aversion » (p. 10) qu'il a pour elle. Plus tard, Pierre, voyant sa fille Lise essuyer une larme de sang qui coule de ses yeux à sa joue et la lécher, détourne «vivement la tête» (p.50) de dégoût tout en durcissant sa mâchoire de colère tandis que sa jambe a un «mouvement nerveux irrépressible » (ibid.). D’ailleurs, le jour où Pierrot décida ne plus rentrer à la maison après le travail, Lise et Maud, ses filles, avaient pleuré « de belles larmes de sang qu'elles ne dissimulaient pas, attablées dans la cuisine », au petit-déjeuner (p.56). Il ressort essentiellement que les pratiques mystiques de ces femmes et de leurs filles sont la cause de l'échec de ces mariages. Ainsi, un jour, rentrant plus tôt que prévu à la maison, le père de Lucie « avait aperçu le bout

\footnotetext{
1 La précarité de l'identité de Lucie joue un rôle primordial dans la construction narrative et structurelle du roman, le récit étant bâti autour d'elle. Cette précarité est à l'image de son effort futile de garder sa famille intacte, tout en raccommodant ses parents. À la fin, elle se retrouvera pratiquement seule, sans son mari, ses filles ou son père chéri.
} 
d'une queue de serpent»(p.128), alors que sa femme, qui s'était transformée pendant ses pratiques occultes, reprenait sa forme humaine. Cet incident, qui crée chez la mère de Lucie une «frénésie d'anéantissement, [...] [une] volonté de [...] châtier, [d]'estourbir, de [...] détruire» (p. 129), son mari ayant "posé son regard là devant quoi il aurait dû fermer les yeux, quand bien même il n'y allait pas de sa faute » (ibid.), c'est-à-dire sa vraie nature mi-animale/mi-humaine, conduira à leur séparation après vingt-cinq années de mariage. Les parents de Lucie illustrent très bien cet échec, qui dans leur cas culmine avec la transformation de son père en escargot par sa mère, bien des années après leur divorce à l'amiable. Quand elles ne s'adonnent pas à leurs pratiques mystiques, les femmes maltraitent les hommes autour d'elles, telle Isabelle qui violente, rudoie, humilie et traumatise son fils Steve, «ce petit abruti qui [lui] gâche la vie » (p. 73) et « grelott[e] de fatigue et d'effroi » en sa présence (p. 24).

\section{Évincement et repositionnement : de l'autre à soi-même}

À travers ces couples mal assortis ou dysfonctionnels, l'auteure présente une image peu flatteuse du mariage et de la famille. Les hommes y mènent une vie devenue si insoutenable que leur seul salut réside en leur fuite et leur abandon du foyer. Cela place l'homme dans une position peu enviable. Il n'est ni pourvoyeur ni défenseur, et cette situation cause une émasculation inacceptable dans un contexte traditionnel et patriarcal tel que celui de La Sorcière. La femme prend ainsi le contrôle, consciemment et violemment comme Isabelle ou la mère de Lucie, ou encore insidieusement et inconsciemment comme Lucie et ses deux filles. Cette prise de contrôle a 
généralement pour corollaire une grande agressivité ${ }^{2}$ et semble inévitable pour combler le vide laissé par le père absent, évincé par sa femme, en raison de la force mentale, psychologique ou encore mystique de cette dernière. Celle-ci devient ainsi le chef, la tête de famille, ce qui fait automatiquement de l'homme autre chose, l'autre, celui qui se trouve en périphérie, le perdu, l'aliéné. Cela est bien illustré dans la famille Matin, où M. Matin a été mis de côté par son fils de neuf ans et sa femme, qui l'ont réduit au simple rang de gagne-pain et de chauffeur. M. Matin, mortifié et exclu de la vie familiale, en vient à se demander si un " homme peut accepter de vivre longtemps comme ça de nos jours » (p.46). Lucie abonde dans le même sens lorsqu'elle excuse l'abandon du foyer par son mari en se remémorant leur vie de famille : «Et Pierrot, qu'aurait-il pu faire d'autre que de s'éloigner de nous, auprès de qui il avait été toujours comme un expatrié contraint et fulminant?» (p.140) En faisant de l'homme l'autre, la femme, désormais, occupe seule le centre névralgique. Dans ce roman de Ndiaye comme dans bien d'autres, l'altérité joue un rôle prépondérant, car il s'agit de créer de nouvelles règles du jeu, un jeu jusque-là contrôlé par l'homme. Cette altérité semble avoir pour objet de nier à l'autre son identité première pour en créer une toute nouvelle.

En effet, dans La Sorcière, la mise en périphérie du père ou du mari est un acte conscient, violent et délibéré de la part de la mère. L'auteure renforce cette absence du père/mari en faisant usage d'une narration réductrice même du concept de

\footnotetext{
2 Cette agressivité pourrait s'expliquer par le fait que les femmes, dans ce roman, ont un ressentiment contre les hommes, qui les empêchent de vivre et leur " gâch[ent] » la vie (p. 73), à l'instar d'Isabelle, qui dit se « crever » (p. 26) pour son fils idiot et imbécile. Isabelle affirme d'ailleurs qu'elle s'en irait toute seule si elle avait beaucoup d'argent (voir p. 72).
} 
père ou de mari. À l'exception de Pierrot, mari de Lucie, et du père de celle-ci, les autres pères ou maris sont rarement évoqués et, quand ils le sont, l'auteure utilise des termes peu flatteurs et de phrases brèves, courtes et souvent laconiques, sans profondeur psychologique ni densité aucune. Ainsi, Isabelle, dans une conversation avec Lucie, décrit son fils et son mari comme «ce petit crampon de Steve et ce bonnet de nuit qu'était son père, [...] deux abrutis » (p. 40). Pour Lucie, le mari d'Isabelle est aussi un homme sans personnalité, dont le «visage n'exprimait rien, ni fatigue ni impatience, que le vide résigné d'une situation habituelle» (p. 25). Cet usage d'économie narrative lorsqu'il s'agit des personnages masculins tend à transformer ceux-ci en simples types caricaturaux. S'il est vrai que le discours de l'instance narrative sur les personnages masculins n'est pas toujours négatif, l'indifférence, la désinvolture et le dédain ${ }^{3}$ qui l'accompagnent, le choix et la parcimonie de moyens descriptifs et discursifs semblent néanmoins avoir pour objet de réduire leurs valeurs et leur importance psychologique. Cette réduction narrative et descriptive se transformera en disparition totale de plusieurs personnages masculins, résultat ciblé de la logique narrative et énonciative «naturelle » du discours du roman. En effet, c'est très justement que Torfi affirme que Marie Ndiaye possède une «maîtrise admirable des ressources de la langue, [une] justesse du vocabulaire et une plasticité extraordinaire de la syntaxe » (2003, p. 69); les choix narratifs ou énonciatifs de l'auteure sont donc parfaitement motivés. Ainsi, quand la mère de Lucie

\footnotetext{
3 C'est avec dédain et désinvolture que Mme Matin, venue récupérer son mari et apprenant que ce dernier vient de s'enfuir en enjambant la haie effrayé à l'idée de rentrer chez lui, dit à son fils : "Alors nous allons l'attendre à la maison, hein, fils? » (p. 56)
} 
transforme son ex-mari en escargot, cette scène est relatée sans émotions ni regrets quelconques, mais plutôt avec détachement, froideur et une banalité frisant le mépris (p. 173174). L'escargot, dans plusieurs cultures, représente la régénération périodique, l'éternel retour, en même temps que la sexualité par analogie à la vulve, réceptacle du sperme (Ronecker, 1994, p. 312-313). Pour Miquel, l'escargot n'est pas apprécié, il est vu péjorativement en raison de son repli sur soi et de la trace visqueuse de son passage (1991, p. 151). Ainsi, en analysant le sort réservé au père de Lucie transformé en un escargot appelé « bestiole », "limaçon » (La Sorcière, p. 173) et transporté dans une vulgaire boîte de bouillon en cubes, et le fait que la boîte sera jetée dans une poubelle dans laquelle le lecteur, à l'instar de Lucie qui se sent responsable du sort de son père, suppose qu'il aura une «fin pitoyable » (p. 182), il est logique de penser que Ndiaye voit en l'escargot un animal inférieur, faible et facilement écrasable. Cette image de l'homme transformé en escargot par la femme est une représentation métaphorique qui réduit l'homme au rang de simple animal inférieur; elle souligne ainsi le peu de valeur accordée aux hommes dans le récit. Ndiaye n'hésite pas à créer des portraits de maris ou de pères pleutres, hommes sans personnalité, irresponsables, incapables, absents et égoïstes. Il n'est pas étonnant que M. Matin inspire « une sorte de répulsion » (p. 51) en racontant ses déboires familiaux à Pierre et à la famille de celui-ci, au lieu de la pitié et la sympathie escomptées. Ce faisant, l'auteure présente les hommes comme des personnes indignes de la sympathie du lecteur, les rendant ainsi responsables du sort qui leur est réservé dans le roman.

La femme bénéficie d'une description plus détaillée, dense, contribuant à lui donner une dimension humaine, 
vivante et parfois chaleureuse, qu'il s'agisse d'Isabelle, antipathique, «ni jolie, ni travailleuse [qui] s'imposait, grasse et forte » (p.17), de Maud et de Lise, "les deux sorcières sans scrupules» (p. 90), «à l'aspect funeste» (p. 98), «féroces » (p. 13), ingrates et méchantes, de la belle-mère de Lucie, femme rondelette et bornée ou encore de la mère de Lucie, femme parée de toutes les qualités. Ce contraste dans la description des femmes et des hommes symbolise la faiblesse, la bassesse et la limitation de l'homme vis-à-vis de la femme, représentée par l'oiseau tout au long du roman. En effet, les femmes du roman se transforment fréquemment en corneilles ou en corbeaux, deux oiseaux qui se confondent dans le symbolisme animal. La corneille représente la déesse de la guerre dans différentes cultures; elle est également l'une des incarnations des Walkyries, chargée de désigner les héros qui vont mourir et les mener au Walhalla. Le corbeau, quant à lui, a un symbolisme négatif et lugubre en Europe, alors qu'en Chine et au Japon, il représente un oiseau solaire qui apporte la lumière, il est un messager divin, il incarne aussi l'amour filial et l'amour familial (Ronecker, p. 116-117). Il est difficile de savoir à quel point ces symbolismes du corbeau et de la corneille sont proches de l'usage que l'écrivaine en fait dans ce roman. L'homme et la femme sont deux êtres différents et diamétralement opposés en tout, sans aucune complémentarité, une véritable antinomie. Marie Ndiaye reformule ainsi la relation hommes-femmes et, par la même occasion, redéfinit l'identité de l'un et de l'autre.

Ce renversement de rôles est important, car il a une portée subversive puisqu'il touche directement et intimement $\mathrm{au}$ système traditionnel du patriarcat, créant le «chaos». Subrepticement, le concept de subversion s'introduit dans l'action narrative et énonciative du roman. En effet, déchu de 
son rôle de chef de famille, institution associée à la stabilité de la communauté, l'homme devient psychologiquement et mentalement émasculé. Traditionnellement et dans l'imaginaire populaire, l'homme est celui qui inspire la crainte à la femme, mais dans ce roman, c'est plutôt l'inverse qui se produit: les femmes inspirent la terreur à leurs maris ou à leurs partenaires. Il n'est donc pas choquant que M. Matin ait une telle frayeur de sa femme qu'il préférera se sauver par la fenêtre dans la nuit plutôt que de rentrer au foyer affronter cette dernière (voir p.56). Ceci est sans aucun doute une des attaques les plus fortes contre l'ordre patriarcal dans ce roman. Les pères et maris fuient ainsi leur maison, « [leur] coin du monde », « [leur] premier univers » (Bachelard, 2009, p. 24) : ils sont perdus à jamais.

Ces comportements qui, à la surface, semblent être le résultat d'actes réalisés ou non par les pères et les maris sont en réalité des actes de violence, violence inscrite dans le fonctionnement interne du roman et liée à sa thématique spécifique, à savoir la pratique de la sorcellerie et son rôle dans la conquête du pouvoir et de la liberté de la femme. La violence, dans ce roman, n'est pas nécessairement physique; elle est surtout psychologique et émotionnelle, mais les effets n'en sont pas moins dévastateurs, tant pour les hommes que pour les femmes, car en faisant violence aux autres, on s'en fait à soimême aussi ${ }^{4}$. Pourtant, il faut tout abandonner pour pouvoir aller de l'avant, passage obligé pour qui veut réussir : « seuls les faibles restent encore entravés par le cordon ombilical de la famille qu'ils ne savent pas couper» (Rabaté, 2004, p. 553).

4 Voir Duffy (2009, p. 927) à propos de la capacité de la race humaine à infliger la souffrance à soi-même et à autrui. 
Dans leur quête identitaire, les femmes veulent réussir dans la vie; elles useront donc de violence pour se désentraver, s'il le faut. Ainsi, Isabelle n'hésitera pas à faire violence sur son jeune fils Steve en lui faisant subir maltraitance et intimidation dans le seul but de se débarrasser de lui afin d'aller se chercher.

\section{Sorcellerie et culture : un choix assumé}

Après avoir savamment introduit un élément subversif en renversant les rôles traditionnellement reconnus aux deux sexes à coups de choix langagiers et d'éléments discursifs judicieux, Ndiaye consolide cette subversion à travers le choix de la pratique de sorcellerie par les femmes comme moyen de renversement de l'ordre établi. Ce choix comme thème de remise en question des normes familiales et sociales est subtilement introduit dans l'élément familial.

L'auteure choisit un moyen de défense et de résistance unique aux femmes; celles-ci possèdent en effet un pouvoir transmis exclusivement de mères en filles. Le thème de la sorcellerie et de sa pratique sont des sujets extrêmement délicats, comme en témoignent non seulement l'histoire occidentale - parsemée de chasses aux sorcières - mais aussi la circonspection et parfois la passion qui accompagnent tout débat ce sur thème.

Qu'est-ce qui a motivé un tel choix? Serait-ce l'ascendance africaine de l'auteure? Rien de moins sûr, si on s'en tient à ses propres paroles. En effet, parlant de son expérience en Afrique, elle affirme :

Je suis allée deux ou trois fois en Afrique, c'est un lieu qui m'intrigue, me fascine aussi, car je sens que j'y suis 
radicalement étrangère. Quand j'y suis et que les gens voient mon nom et la couleur de ma peau, ils pensent que je suis des leurs. Or, par mon histoire, c'est faux. J'ai souvent rencontré des Français qui ont été élevés en Afrique et qui sont plus africains que moi. Alors qu'eux, en Afrique, dans le regard des autres, ils restent étrangers... Ironiquement, c'est en France que je peux paraître étrangère. (dans Inrocks, 2009)

Certains critiques avancent néanmoins que ce choix est influencé par l'Afrique, du fait de l'ascendance africaine de l'écrivaine. Ainsi, Colette Sarrey-Strack avance que Ndiaye a puisé dans le conte africain (voir 2002, p. 64) en choisissant le thème de la sorcellerie ${ }^{5}$ comme solution aux problèmes de la famille et comme outil pour permettre aux femmes de retrouver leur identité et d'accéder ainsi au pouvoir et à la liberté, ce qui semble être en porte-à-faux avec les dires de l'auteure. En effet, celle-ci déclare :

J'ai été élevée uniquement par ma mère, avec mon frère, en France. Pas par mon père, avec qui je n'ai jamais vécu, et que je ne suis pas allée voir en Afrique avant l'âge de 22 ans. J'ai été élevée dans un univers $100 \%$ français. Dans ma vie, l'origine africaine n'a pas vraiment de sens [...]. (ibid.)

La Sorcière est situé dans une ville provinciale typiquement française, dans un milieu petit-bourgeois franco-français. Apporter un élément étranger ou extérieur pour trouver une solution à un problème interne est-il nécessaire? D'ailleurs, l'écrivaine, par le choix de cette ville essentiellement française comme lieu d'action de son roman et de la sorcellerie comme thème, établit un pacte ou un contrat avec son lecteur. Il est clair que les lecteurs potentiels de Marie Ndiaye possèdent une certaine perception des choses et des idées fortement

5 Barnet (2009, p. 232) affirme que «Marie Ndiaye a repris de façon obsessionnelle » le motif de la sorcière. 
influencée par la littérature mais aussi par l'environnement historique, sociologique, politique et religieux dans lequel ils ont été socialisés. En effet, la littérature, selon Canvat, « renvoie tout à la fois à ces composantes textuelles que sont les propriétés matérielles, pragmatiques et structurelles du texte, l'ensemble de ses conventions esthétiques et formelles, l'espace hypertextuel et intertextuel où jouent des mécanismes d'imitation et de transgression » (1992, p. 42). C'est dans un tel contexte que s'inscrit également l'œuvre de l'écrivaine sur le plan tant thématique que narratif. Ses choix ciblés jouent le rôle «d'opérateur de cadrage qui permet la reconnaissance / identification du texte et qui facilite donc sa compréhension; il contribue à informer le texte (c'est-à-dire à lui donner forme et sens) » (Canvat, 1999, p.115). On suppose ainsi que le lecteur saura comprendre le texte sans trop de difficultés. Mais ceci sera-t-il le cas?

Un regard sur la conception de la sorcellerie dans la culture africaine révèle sans équivoque que le choix de Ndiaye est plutôt motivé par l'histoire de la sorcellerie en Occident et non pas en Afrique. En effet, dans le contexte africain, tous, hommes comme femmes, peuvent être également accusés de pratique de sorcellerie et châtiés en conséquence. Ainsi, le point de vue de Colette Sarrey-Strack découle d'une méconnaissance de la conception de la pratique de la sorcellerie en Afrique6, Sarrey-Strack faisant une transposition culturelle erronée. Dans la culture judéo-chrétienne occidentale, par contre, la pratique

\footnotetext{
6 Ce point de vue, qui n'est pas unique à Sarrey-Strack, conditionnera inévitablement la lecture, la réception et la compréhension de La Sorcière. Pour Little (2000, p. 362), par contre, la couleur de la peau de Ndiaye ne conditionne pas nécessairement son écriture: elle n'est pas vraiment une écrivaine noire, si on s'en tient à certains de ses choix.
} 
de la sorcellerie est historiquement liée à la personne de la femme $^{7}$ et renvoie à l'association supposée de la femme et du diable, à l'image du lien biblique entre Ève et le serpent. Cette pratique, dans le monde judéo-chrétien, est fortement réprimée par l'église, qui condamne toute personne accusée de sorcellerie à être brûlée vive sur le bûcher afin de purifier son âme. L'influence de la tradition biblique chrétienne est ici évidente. La pratique de la sorcellerie en Occident est donc vue comme une abomination, comme un péché mortel passible d'un châtiment des plus atroces : être brûlé sur la place publique pour servir d'exemple et décourager de telles pratiques. L'intention de l'auteure semble indéniable ici : si la sorcellerie a été une pratique occulte reprouvée aux siècles passés et punie par un châtiment atroce, chez Marie Ndiaye, celles qui la pratiquent reçoivent une absolution totale, la sorcellerie devenant une pratique salvatrice qui désentrave les femmes.

Ce choix original du rôle de la sorcellerie constitue une continuité dans le combat des femmes commencé depuis plusieurs siècles et une (re)mise en valeur de cette pratique violemment reprouvée par l'Église et la croyance populaire. Pourtant, ce choix délibéré a un rôle d'une grande portée symbolique, car il représente une subversion du concept même de sorcellerie par sa référence à l'histoire et à l'imaginaire populaire. La pratique de la sorcellerie devient ainsi l'outil principal dans la quête identitaire de la femme et dans la revendication de son droit à la liberté mentale, émotionnelle et même financière, c'est-à-dire de son bonheur, ce qui représente une subversion poussée à l'encontre du patriarcat. Inspiré par

\footnotetext{
7 Ceci est attesté par les expressions encore en vigueur, « faire la chasse aux sorcières », et son équivalent anglais, « to go on a witch hunt ».
} 
Ricœur, Michel Thérien soutient que «l'œuvre littéraire transmet une somme incalculable de réflexions, de pensées, d'expériences, de sagesse, en attente d'être partagées, d'être critiquées, dans une réception dialogique » (1992, p. 78). Par le choix de la sorcellerie comme thème libérateur capable de conduire à la résolution identitaire, l'écrivaine force le lecteur à ouvrir un débat culturel, personnel, historique et surtout métaphysique sur ce concept, dans le but de l'amener à une certaine émancipation réflexive. En effet, Ndiaye utilise cette même sorcellerie reprouvée et condamnée et la pare de pouvoirs libérateurs pour les femmes; subversif en lui-même, elle transforme et redéfinit le concept de sorcellerie en lui attribuant des qualités positives, bénéfiques et utiles aux femmes. Cet acte même de libération est également subversif, car la femme n'a plus d'utilité pour l'homme, elle poursuit la quête et la réalisation de son identité à travers la pratique de la sorcellerie. Étant donné que, dans le monde de La Sorcière, ce domaine est contrôlé uniquement par la femme, l'homme, exclu, perd automatiquement le contrôle sur sa femme, sur son foyer et sur sa communauté ; il est évident que ce que l'homme perd en contrôle, la femme le gagne en liberté et en pouvoir, les deux allant de pair, ce qui renforce la rébellion contre l'ordre établi. S'il est vrai que quelques hommes furent accusés de sorcellerie dans l'histoire occidentale, la très grande majorité des personnes brûlées vives sur le bûcher pour pratique de sorcellerie sont assurément des femmes. L'auteure utilise donc un moyen interne, qui se trouve au sein même de la culture dans laquelle elle évolue, c'est-à-dire la culture française, pour trouver une solution au problème débattu. 


\section{Sorcellerie et champ conceptuel}

En créant des femmes libérées en grande partie ou même uniquement grâce à leurs pratiques de la sorcellerie, l'auteure a réussi à recentrer le terme de "sorcellerie » et à redéfinir le champ conceptuel qu'il recouvre. À cause des pouvoirs que leur confère la sorcellerie, les femmes seront capables de mener à bien leur vie, loin de leurs maris, quoique leurs pratiques de sorcellerie causent également la destruction de leurs familles. Jordan souligne d'ailleurs que «La Sorcière stands as Ndiaye's most potent statement about feminine identity» (2004, p. 172) ${ }^{8}$. Il semble en outre que l'auteure veuille créer une identité chez ces femmes ou plutôt faire ressortir une dimension qu'elles posséderaient déjà, mais dont elles ne seraient plus conscientes, une identité oubliée. Cette identité constituera la clé de leur liberté, celle qui ouvrira leur mémoire collective enfouie au fond d'elles-mêmes, afin d'empêcher un effacement progressif de cette mémoire. Forger cette identité doit consister à conjuguer deux aspects : consolider en même temps l'aspect individuel et collectif de l'identité. Il n'est donc pas étonnant que Lucie, la narratrice, croie voir en toute femme une sorcière potentielle, chaque femme étant différente et semblable à l'autre, qu'il s'agisse d'Isabelle qui se serait transformée en corneille à la fenêtre de ses parents à Paris ou toutes ces jeunes étudiantes à l'Université Féminine de la Santé Spirituelle d'Isabelle 0. (p.153) ${ }^{9}$, ou même Lili, la sœur de Pierrot, qui ressemble étrangement à ses deux nièces, Lise et Maud, ces deux dernières s'étant envolées en abandonnant leur

\footnotetext{
8 Je traduis : «La Sorcière est la plus puissante affirmation, chez Ndiaye, de l'identité féminine. »

9 Université enseignant les pratiques de la sorcellerie libératrice, réservée exclusivement aux filles.
} 
mère Lucie (p. 136) une fois leurs pleins pouvoirs de sorcières acquis, c'est-à-dire leur résolution identitaire accomplie.

$\mathrm{Au}$ début du roman, on rencontre des couples vivant ensemble avec leurs enfants, bon gré, mal gré. À mesure que le récit se déroule, les couples se désagrègent et, vers la fin du roman, toutes les familles sont séparées, à l'exception notable de la mère de Lucie et de son nouveau compagnon, bien que ceci ne soit plus qu'une question de temps. Le mariage, métaphore de la prison, est détruit et les femmes sont libérées : Lucie, Isabelle, Mme Matin, Maud et Lise et leur grand-mère.

Il convient ainsi de se demander si cette introduction du thème de la sorcellerie est simplement un moyen de subversion de l'ordre patriarcal établi ou plutôt une subversion de ce que la sorcellerie est censée accomplir dans ce roman même. En effet, les pratiques de sorcellerie créent certaines attentes dans l'imaginaire des lecteurs, et celles-ci ne seront pas nécessairement comblées. Ndiaye montre que la seule pratique de la sorcellerie ne suffit pas pour se libérer, pour retrouver son identité ou pour trouver le bonheur; il faut surtout bien la pratiquer, à l'instar de la mère de Lucie, « une sorcière parfaite et intègre » (p.54) qui a trouvé cette paix et cet équilibre que procure la vraie et bonne pratique de la sorcellerie.

Une comparaison de toutes les femmes qui ont été mêlées de près ou de loin à des pratiques de sorcellerie révèle que leur degré de liberté et de satisfaction personnelle est proportionnel à leur degré de résolution identitaire, lequel est tributaire de leur engagement et de leur perfection ${ }^{10}$ dans cette pratique. En

10 Lucie est d'ailleurs émerveillée par la "perfection de [ses] filles », dans leurs pratiques, à peine initiées (voir p. 30). 
d'autres termes, tout dépend de leurs compétences. Ainsi, les deux jumelles, Maud et Lise, libérées de toute entrave, s'envolent dans les airs pour ne plus revenir, la métaphore de l'oiseau qui s'envole renvoyant dans l'imaginaire collectif à la liberté et potentiellement au bonheur. Leur grand-mère, malgré certaines actions pourtant reprochables et choquantes, semble épanouie : elle est « désormais plus heureuse, plus satisfaite et plus fidèle à sa vraie nature » (Cottille-Foley, 2006, p. 84), ayant enfin pleinement assumé sa véritable identité de sorcière.

Pourtant, Isabelle, riche propriétaire de l'Université Féminine de la Santé Spirituelle, ne semble pas heureuse, tout comme Lucie, professeure de sorcellerie de ladite université, n'est ni plus heureuse ni plus épanouie que durant son mariage, ce qui constitue un échec de leur quête identitaire. Une analyse de cette situation montre que la différence primordiale réside dans le fait que Maud, Lise et leur grand-mère sont des sorcières accomplies, qui ne montrent aucune émotion et ne font aucun cas des faits et gestes autour d'elles. Or, Lucie ${ }^{11}$ est une sorcière aux pouvoirs approximatifs qui transporte un lot d'émotions de tous genres, et Isabelle, la presque-pas-sorcière «peu aimable, acrimonieuse et rusée » (p. 22), doit également canaliser toutes sortes de sentiments, à commencer par sa monumentale fraude que constitue son Université; les deux femmes se révèlent incapables de gérer efficacement leurs émotions. La lecture qui en ressort montre qu'il faut pouvoir transcender ses émotions et parfaire ses compétences. Leur introspection, censée conduire à une redéfinition de leur identité féminine individuelle, mais surtout collective, positive

11 Duffy (p. 906) trouve Lucie «infantile... very human». Je traduis: « infantile... trop humaine». 
et épanouie, n'a pas abouti. À l'instar de cette introspection ratée, les vies médiocres de ces deux femmes reflètent l'absence de bonheur dans leur existence et mettent à jour une "vérité » presque banale: la pratique de la sorcellerie ne conduit à la résolution identitaire et, par conséquent, au bonheur et à la liberté que si on s'y investit entièrement. L'incapacité des femmes à se débarrasser de tous sentiments et émotions explique leur faillite. Leur seule consolation serait qu'elles sont libérées des entraves que seraient enfants et maris ; de ce point de vue, elles ont progressé dans leur lutte vers la libération et le bonheur.

La redéfinition du concept de sorcellerie et des connotations qui lui sont attachées subit également une reconceptualisation. Tout au long du roman, Ndiaye construit tout un discours autour de la sorcellerie et crée ainsi des attentes précises de la part du lecteur. Néanmoins, le dénouement ou plutôt le non-dénouement du roman remettra en cause, et de façon subversive, le concept de sorcellerie et les attentes si finement et patiemment tissées par l'auteur. Pour nous, cette situation permet une pluralité de lectures et d'orientations plutôt qu'une "confusion interprétative», comme l'affirme Cottille-Foley (2009, p. 548). Dans un genre littéraire "typique» et "normatif», il y a forcément des conventions et des contraintes narratives, et celles-ci sont souvent transgressées. Ndiaye ne rentrant dans aucun genre "classique», son écriture n'obéit à aucune convention. Cet usage antiphrastique des attentes «logiques»du lecteur qui cause un brouillage des codes à plusieurs niveaux et donne lieu au non-dénouement constitue d'ailleurs aussi une subversion. C'est en effet la subversion ultime, car l'entité auctoriale, après avoir pratiquement amené le lecteur à accepter ses règles du 
jeu, les brise elle-même ${ }^{12}$. Il est évident que l'écrivaine va à l'encontre des stéréotypes du genre ou de la culture qui, selon Hamon, assurent «un "minimum lisible" [...] pour définir un horizon d'attente et de prévisibilités qui détermineront pour le lecteur des lignes de moindres résistance et de frayage prédéterminés » (1974, p.116). Il est difficile de situer avec précision Marie Ndiaye dans un courant ou une école littéraire. Cette difficulté à classer l'écrivaine se manifeste ici à travers sa technique d'écriture et sa construction narrative, qui laissent la porte ouverte quant au rôle précis de la sorcellerie. Et ceci, d'autant plus que cette pratique ne peut être prouvée empiriquement tout comme elle n'est pas compréhensible au lecteur typique, comme l'affirme Asibong (2010, p.354). Ce texte, à l'instar de la majorité des textes de Marie Ndiaye, peut donc sans hésitation être classé comme "postmoderne », si l'on s'en tient au brouillage systématique des codes et à son hétérogénéité. Dans une interview, Ndiaye, parlant de la fin d'un de ses romans, déclare : «Je ne propose aucune fin morale. Aucune conclusion édifiante. Je dirais plutôt qu'il s'agit d'une représentation exagérée de ce je vois " (dans Pellegrini, 2004, p. 44); cette formule pourrait bien s'appliquer aussi à $L a$ Sorcière.

Marie Ndiaye ne rentre dans aucun moule littéraire : elle n'est pas vraiment une écrivaine africaine, elle n'est pas considérée non plus pleinement comme une écrivaine française, ni comme une écrivaine seulement francophone. Asibong explique cette

12 Lucie elle-même en vient à douter de ses pouvoirs de sorcière (voir p. 169). 
situation en affirmant que Ndiaye, quel que soit son degré d'assimilation, ne peut prendre pour acquis une non-identité transcendante et postmoderne en raison de sa position de «metic» dans la société française (p. 350). La Sorcière peut ainsi être vue comme une métaphore ayant pour objet d'amener le lecteur à une émancipation réflexive. En effet, le thème de "sorcellerie » et son exploitation veulent dépoussiérer la compartimentation des pratiques, des acceptions, des idées reçues et entretenues.

Si la femme, dans ce roman, réussit à vaincre sa peur, la peur du qu'en dira-t-on, la peur de ne plus être sous la domination financière de l'homme, la peur de se reconnaître et de se proclamer "sorcière ", c'est grâce à la résistance qu'elle oppose à l'ordre établi et son refus de demeurer dans le carcan patriarcal; c'est ce choix délibéré qui conduira à son émancipation, seul moyen de (re)trouver une identité saine.

\section{Bibliographie}

AsIBONG, Andrew. (2010), « Radically Fantastical: The Politics of the Truth-Event in the "metic" novels of Mohammed Dib and Marie Ndiaye», Contemporary French and Francophone Studies, vol. 14, no 4, p. 349-356.

BACHElard, Gaston. (2009), La Poétique de l'espace, Paris, PUF.

BARNET, Marie-Claire. (2009) "Espèce de vilaine fée" ou comment les esprits revinrent à Ève Brulard: la sorcière dans l'œuvre de Marie Ndiaye », dans Margaret-Anne Hutton (dir.), Redefining the Real. The Fantastic in Contemporary 
French and Francophone Women's Writing, Oxford, Peter Lang, coll. « Modern French Identities », p. 224-244.

CAnvaT, Karl. (1992), "Interprétation du texte littéraire et cadrage générique », Pratiques, Paris, № 76.

- (1999), Enseigner la littérature par le genre. Pour une approche théorique et didactique de la notion de genre littéraire, Bruxelles, de Boeck-Duculot.

Cottille-Foley, Nora. (2009), «Optique fantastique, traitement de la photographie et transgression des limites du visibles chez Marie Ndiaye », Contemporary French and Francophone Studies, vol. 13, no 5, p. 547-554.

- (2006), « Postmodernité, non-lieux et mirages de l'anamnèse dans l'œuvre de Marie Ndiaye », French Forum, vol. 31, no 2, p. 81-94.

DufFy, Jean H. (2009), "Liminality and Fantasy in Marie Darrieussecq, Marie Ndiaye and Marie Redonnet», MLN, vol 124, p. 901-928.

Hamon, Philippe. (1974), " Note de la notion de norme et de lisibilité en stylistique », Littérature, Paris, no 14. p. 114122.

LES INROCKS. (2009) L'écrivain Marie Ndiaye aux prises avec le monde, 30 août, <http://www.lesinrocks.com/2009/08/30/actualite/lecriva in-marie-ndiaye-aux-prises-avec-le-monde-1137985/>.

Jordan, Shirley, A. (2004), Contemporary French Women's Writing. Women's Visions, Women's Voices, Woment's Lives, Oxford, Peter Lang. 
LitTLE, J. P. (2000), "The Legacy of Medea: Mariama Bâ, Un chant écarlate and Marie Ndiaye, La Femme changée en bûche », Modern Language Review, vol. 95, nํ 2, p. 362-373.

MiQuel, Dom Pierre. (1991), Dictionnaire symbolique des animaux. Zoologie mystique, Paris, Léopard d'Or.

NDIAYE, Marie. (2003), La Sorcière, Paris, Minuit.

-. (1994), Un temps de saison, Paris, Minuit.

-. (1990), En famille, Paris, Éditions de Minuit.

-. (1989), La femme changeÏe en bûche, Paris, Minuit.

-. (1985), Quant au riche avenir, Paris, Minuit.

Pellegrini, Galli Rosa. (2004), «Marie Ndiaye : De l'abandon à la (ré)appropriation. Thèmes et techniques romanesques", dans Rosa Galli Pellegrini (dir.), Trois études sur le roman contemporain. Marie Ndiaye, Sylvie Germain, Michel Chaillou, Paris, Scena-Presses de l'Université de Paris-Sorbonne, p. 11-49.

RABATE, Dominique. (2004), " “Où est ma famille?” : la violente étrangeté de Marie Ndiaye », dans Bruno Blanckeman, Marc Dambre et Aline Mura-Brunel (dir.), Le Roman français au tournant $d u$ XXIe siècle, Paris, Presses Sorbonne Nouvelle, p. 549-561.

RoneCKER, Jean-Paul. (1994), Le Symbolisme animal. Mythes, croyances, légendes, archétypes, folklore, imaginaire, St-Jeande-Braye, Dangles.

SARREY-STRACK, Colette. (2002), Fictions contemporaines au féminin, Paris, L'Harmattan. 
THÉRIEN, Michel. (1992), « La lecture littéraire au secondaire : le point de vue de la didactique », Tangence, no 36, p. 75-84.

TuliniUS, Torfi H. (2003), «Relations proches», L'Atelier du roman, vol. 35, p. 68-75.

\section{Résumé}

Quels moyens utilise la femme pour vaincre la peur et pour résister aux pressions d'une société très largement encore patriarcale? La construction d'une mémoire collective au féminin est-elle une solution viable? Dans La Sorcière, Marie Ndiaye, écrivaine de la nouvelle génération, approche ce thème de la résistance par un processus que l'on pourrait qualifier de «ré-appropriation identitaire» et qui conduirait à l'affranchissement et à la liberté de façon très particulière. Son outil de choix pour les femmes : la pratique de la sorcellerie.

\section{Abstract}

What means do women possess in conquering fear while resisting pressure from a society still entrenched in patriarchy in Marie Ndiaye's La Sorcière? Does the solution lie in constructing a female collective memory? Ndiaye, a writer of the new generation, deals with the theme of resistance by using a process that can be termed 'identity re-appropriation' a process meant to bring them emancipation and freedom in a very special way. Her choice tool is the use of witchcraft by women. 